\title{
Hydrogel Microspheres I. Preparation of Monodisperse Hydrogel Microspheres of Submicron or Micron Size
}

\author{
Haruma Kawaguchi, ${ }^{*}$ Megumi Kawahara, Nozomi Yaguchi, \\ Futoshi Hoshino, ${ }^{\dagger}$ and Yasuji OHTSUKA \\ Department of Applied Chemistry, Faculty of Science \& Technology, \\ Keio University, 3-14-1 Hiyoshi, Kohoku-ku, Yokohama 223, Japan
}

(Received May 6, 1988)

\begin{abstract}
Precipitation polymerization of acrylamide derivatives in water resulted in the formation of monodisperse submicron particles. Repetitive seeded polymerizations using the product of precipitation polymerization or precipitation polymerization in ethanol-water media gave larger monodisperse particles. Especially, the latter was very effective for the preparation of micro-size particles.

KEY WORDS Hydrogel / Microsphere / Precipitation Polymerization / Seeded Polymerization / Swelling /
\end{abstract}

Hydrogel is a subject of great interest in these days due to its characteristic swellingdeswelling behavior in response to changes of temperature, $\mathrm{pH}$, medium components, etc. and applications based on this character in medical, agricultural, and industrial fields. In many cases, quick and uniform responses are necessary for successful applications. The rate of response is inversely proportional to the square of its size. ${ }^{1}$ Therefore, hydrogels are often used in small pieces, fine filaments, or thin films. Small pieces of hydrogels are usually prepared by crashing a bulk of gel or by inverse suspension polymerizations. ${ }^{2,3}$ The particles given by inverse suspension polymerization have a diameter in the range of 10 to $100 \mu \mathrm{m}$ and not necessarily monodisperse.

Recently, smaller and monodisperse microspheres were prepared by a novel precipitation polymerization by Pelton and Chibante. ${ }^{4}$ They polymerized $N$-isopropyl acrylamide with $N, N^{\prime}$-methylenebisacrylamide in water at $70^{\circ} \mathrm{C}$. As the polymerization temperature was much higher than the lower critical solution temperature (LCST) of resulting polymer, $c a$. $35^{\circ} \mathrm{C}$, the reaction accompanied precipitation of polymers and produced a dispersion of polymer particles with submicron diameter. Some other acrylamide derivatives also give polymers with their specific LCSTs. For example, the polymers of acryloyl pyrrolidine and acryloyl piperidine have their LCSTs at $50^{\circ} \mathrm{C}$ and $5^{\circ} \mathrm{C}$, respectively. We have used such monomers with styrene to prepare latex particles having shells composed of them, which exhibited reversible expansion-shrinkage with changing temperature. ${ }^{5,6}$ The precipitation polymerization of these acrylamide derivatives was found to result in the formation of monodisperse submicron hydrogel microspheres.

The most suitable size of hydrogel microspheres in their applications differs from case to case. Submicron-size might be too small to treat in many cases. The smaller the particles

\footnotetext{
† Present address: Mitsui Toatsu Chemicals Ltd., Central Research Institute.
} 
are, the smaller the ratio of volume to surface becomes, and consequently the less the utility value of their reversible expansion-shrinkage becomes. These drawbacks may be eliminated by enlarging the particles to micron-size. Therefore, the development of preparative methods for monodisperse hydrogel microspheres of micron-size is a subject worth challenging. In this paper, some precipitation polymerizations and seeded polymerizations were examined to prepare these hydrogel microspheres.

\section{EXPERIMENTAL}

\section{Materials}

Two kinds of $N$-substituted acrylamides, acryloyl pyrrolidine (APr) and acryloyl piperidine (APp), were kindly given by Mitsui Toatsu Chemicals Ltd. They were distilled at $89^{\circ} \mathrm{C}(0.5 \mathrm{mmHg})$ and $85^{\circ} \mathrm{C}(0.05 \mathrm{mmHg})$, respectively. $\quad N, N^{\prime}$-methylenebisacrylamide (MBAAm) was recrystallized from methanol. The initiators used were $N, N^{\prime}$-azobis(amidinopropane)dihydrochloride (APH, Wako Chemicals Co.) and potassium persulfate (KPS, Taisei Chemicals Co.), the latter of which was recrystallized from water whereas the former was used without further purification.
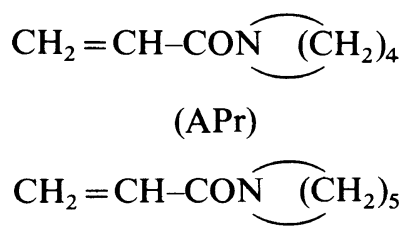

(APp)

\section{Precipitation Polymerization}

The polymerization condition was decided by referring to the Pelton's method. ${ }^{4}$ In standard recipes, $1 \mathrm{~g}$ APr or $3 \mathrm{~g}$ of APp and 0.1 or $0.3 \mathrm{~g}$ MBAAm were dissolved in $195 \mathrm{~g}$ distilled water. Polymerization was carried out in a $300 \mathrm{ml}$ round bottom flask equipped with a stirrer, a condenser, and nitrogen inlet and outlet. The reaction was started by adding $5 \mathrm{ml}$ of $0.2 \%$ aqueous solution of the initiator at $70^{\circ} \mathrm{C}$ under nitrogen with gentle stirring. The polymerization was continued in all cases for $1.5 \mathrm{~h}$ unless otherwise noted. To prepare larger microspheres, ethanol was added in the medium up to $5 \%$.

\section{Seeded Polymerization}

The first seeded polymerization was carired out by adding $1 \mathrm{~g}$ APr and $0.1 \mathrm{~g}$ MBAAm to the product from above-mentioned polymerization. The following seeded polymerizations were repeated in the same manner.

\section{Measurements}

The contours of the microspheres were observed and their sizes measured by transmission and scanning electron microscopy. The hydrodynamic size was determined by photon correlation spectroscopy using LPA3000 and -3100 systems (Ohtsuka Electrics Co.). The partition of monomer between polymer precipitate and medium was determined by a UV-spectrometric measurement of monomer remaining in the medium after the equilibrium absorption.

\section{RESULTS AND DISCUSSION}

\section{Precipitation Polymerization in Water}

Both precipitation polymerization of $\mathrm{APr}$ and APp in water resulted in the formation of submicron microspheres but the character of their products was quite unique for each. The transmission electron micrographs are shown in Figure la and 1b. Although the samples for electron microscopy were prepared in the same condition, the poly-APr and poly-APp microspheres in the figures differed completely from each other. The former had obscure contour and thin image whereas the latter had clear and thick ones.

This came from differences in the extent of swelling of two kinds of microspheres at the temperature at which the samples were pre- 

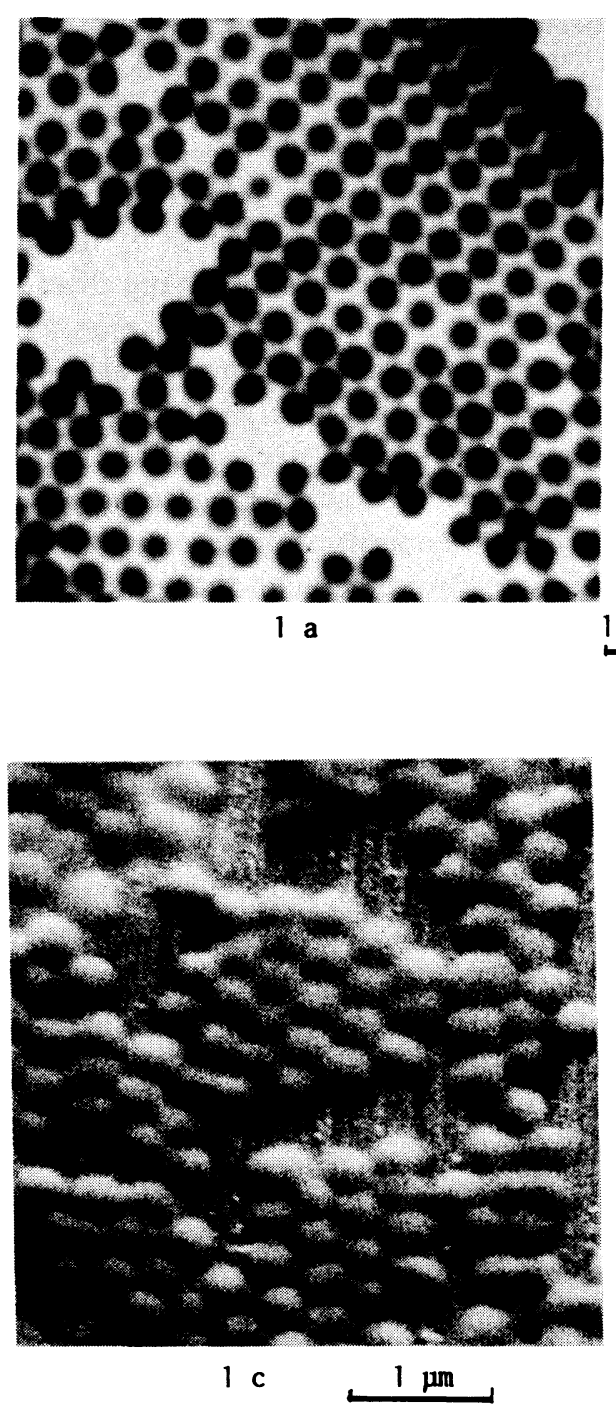

pared for electron microscopy. In the sample preparation, the diluted dispersions of microspheres were put onto collodion membranes on sheet meshes and dried at room temperature lower than the LCST of poly-APr but higher than that of poly-APp. Poly-APr microspheres hold a large amount of water at the room temperature and the drying of them gave the partially shrank microspheres, perhaps having an infinite number of fine voids, which were observed as obscure and thin particles on the transmission electron micrograph. The scan-

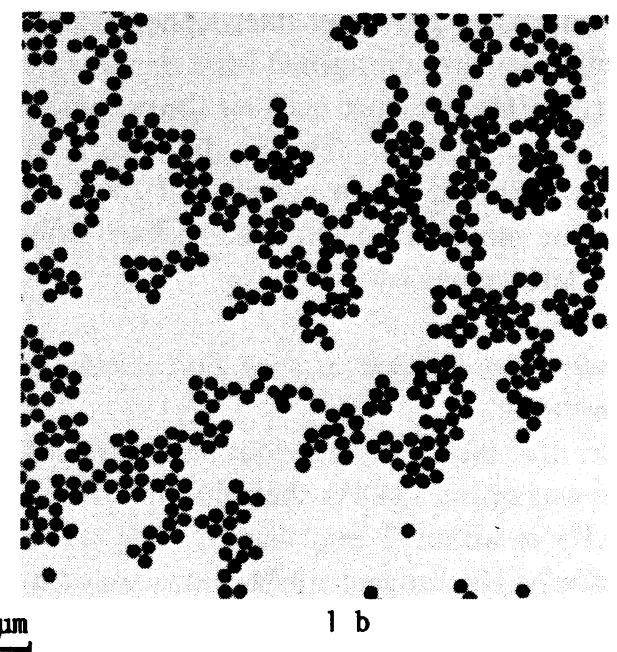

Figure 1. Electron micrographs of hydrogel microspheres of poly(acryloyl pyrrolidine) (APr) and poly(acryloyl piperidine) (APp) prepared by precipitation polymerizations at the standard recipes. la and $1 \mathrm{c}$, poly-APr; 1b, poly-APp; $1 \mathrm{a}$ and $1 \mathrm{~b}$, transmission electron micrographs; lc, scanning electron micrograph.

ning electron micrograph of the same sample revealed that the poly-APr microsphere (Figure 1, 1c) was slightly deformed or flattened during the drying.

The occurrence of an orderly array of polyAPr particles on the micrograph la must be evidence for the monodispersity of hydrated microspheres at the closedly packed state. Actually, the distribution curve for the hydrodynamic diameter of microspheres measured by photon correlation spectroscopy was a sharp uni-modal one. 
In contrast to poly-APr microspheres, polyAPp microspheres contained little or no water at the room temperature because the polymer's LCST was about $5^{\circ} \mathrm{C}$. This is the reason for high-contrast and sharp contour of their micrographic image similar to that of hard polystyrene latex particles.

\section{Dependence of Particle Size on Polymerization Conditions}

First, the effect of the monomer concentration was examined. As the solubility of APr and APp is about 2 and $4 \mathrm{~g}$ in $200 \mathrm{~g}$ water, respectively, the range examined was very limited. The increase in the amount of APr from $1 \mathrm{~g}$ to $2 \mathrm{~g}$ and that of APp from $3 \mathrm{~g}$ to $4 \mathrm{~g}$ resulted in the formation of $15 \%$ and $20 \%$ larger microspheres but they were less stable than those prepared by standard recipes.

Next, the effect of the amount of MBAAm was examined and the results are shown in Figure 2. There were almost opposite dependences of size on the amount of MBAAm

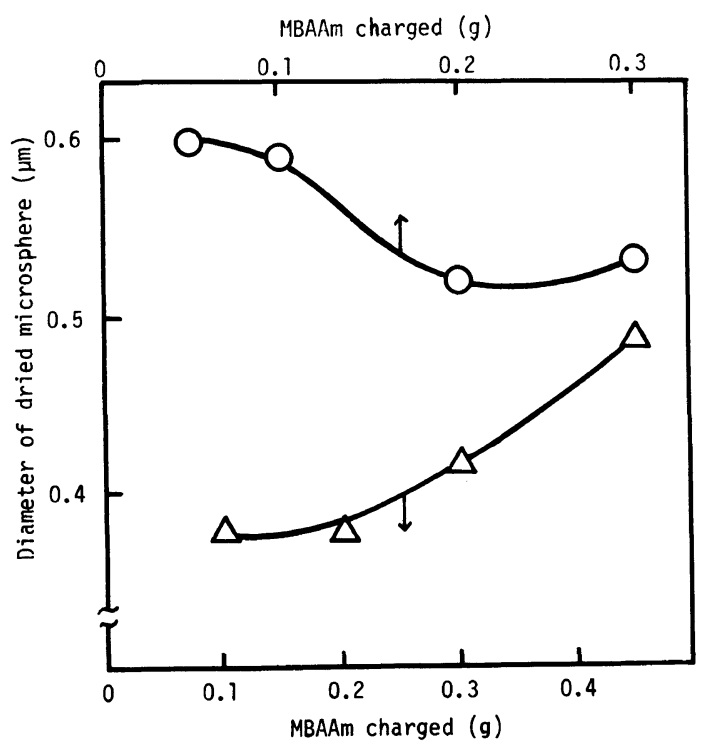

Figure 2. Influence of methylenebisacrylamide (MBAAm) concentration on the size of poly(acryloyl pyrrolidine) and poly(acryloyl piperidine) microspheres. $O$, poly-APr; $\triangle$, poly-APp. Recipe was the same as standard one except for MBAAm concentration. between APr and APp systems.

This can be explained as follows: MBAAm has two conflicting effects on the size of microspheres in aqueous precipitation polymerization. One of them is a tightening effect which makes the microsphere small by dense crosslinking. The other is a dissolving effect, that is, an effect to make the copolymer more hydrophilic and retard the precipitaion, resulting in a small number of large microsphers. In APr system, the former effect appeared first and the latter started to affect the size only at higher MBAAm concentration, because APr itself is fairly hydrophilic. On the contrary, in the much less hydrophilic APp system, the latter played a role even at a low MBAAm concentration.

Two kinds of initiators were used in this study. APH gave more stable dispersion than KPS did. Therefore, APH was used exclusively in the following study. The increase in the amount of APH brought about a decrease in the size of poly-APr and poly-APp microspheres. This was attributed to the formation of a large number of nuclei caused by a high rate of radical supply in high APH concentration.

As shown above, it was difficult to prepare microspheres having micro-size by adding a small change in the reaction conditions. Therefore, some other methods were tried to make this possible.

\section{Seeded Polymerization of APr in Water}

Repetitive seeded polymerizations are often adopted for enlargement of microspheres. Seeded polymerization was carried out using poly-APr microspheres, as the first seed, prepared by the precipitation polymerization. As mentioned above, tight crosslinking suppresses the enlargement of seeds, and the amount of MBAAm was decreased to a half of that in the standard recipe, that is, the ratio of MBAAm to APr was kept to be 0.05 in a series of seeded polymerization.

Successful results were obtained till the fifth 


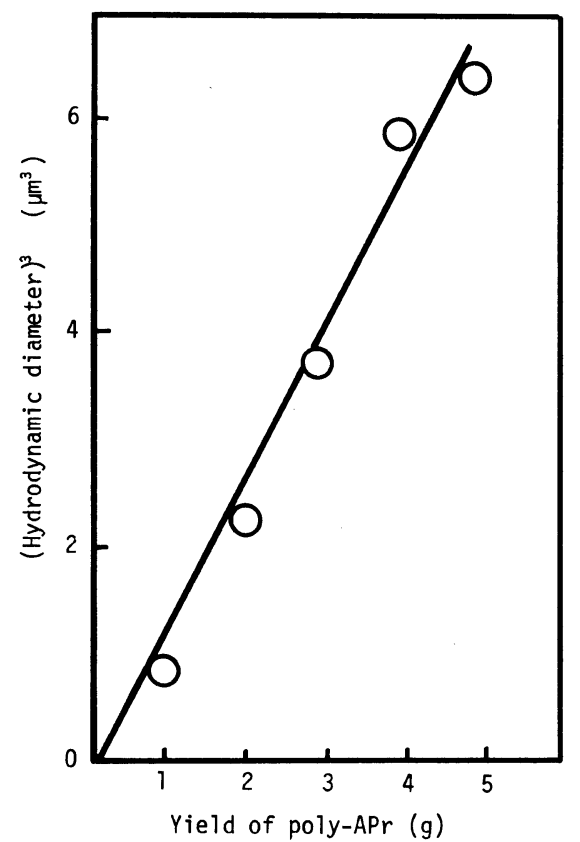

Figure 3. Size of poly(acryloyl pyrrolidine) microspheres obtained by repetitive seeded polymerization as a function of polymer yield. Every polymerization was carried out by adding $1 \mathrm{~g}$ APr and $0.05 \mathrm{~g}$ MBAAm to previously-formed seed system at $70^{\circ} \mathrm{C}$ for $1.5 \mathrm{~h}$.

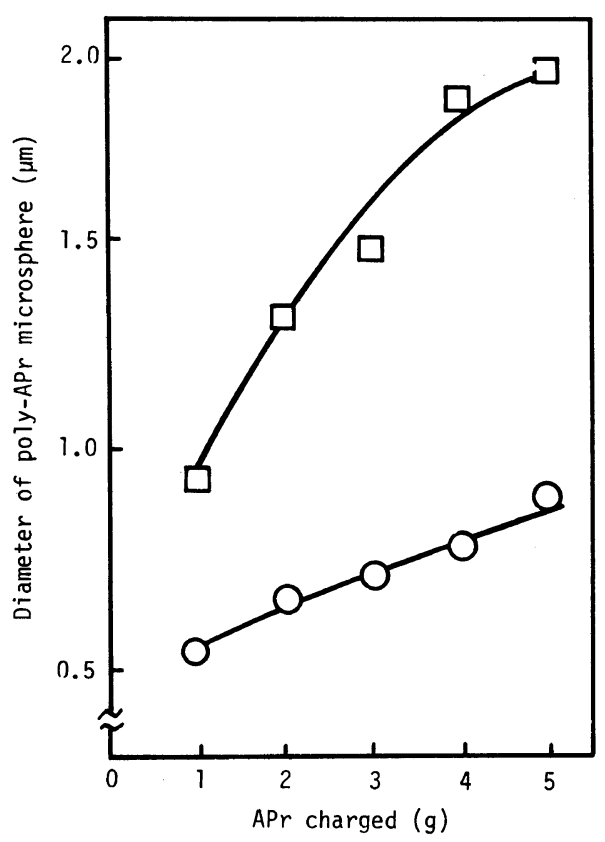

Figure 4. Size of poly(acryloyl pyrrolidine) microspheres in the dry and hydrodynamic states. $\bigcirc$, diameter of dried particles; $\square$, hydrodynamic diameter. seeded polymerization. Namely, it was apparent from the observation of electron micrographs of poly-APr microspheres after each seeded polymerization that the particles were enlarged with reasonable increments at each step, keeping monodispersity without forming new small microspheres. The proportionality of (diameter) ${ }^{3}$ with polymer yield shown in Figure 3 proved the normal progress of repetitive seeded polymerization.

The aim to get microspheres of micron-size in their dry state, however, was not attained by repeating the seeded polymerization (Figure 4) because the sixth seeded polymerization gave some coagulum. Flory deduced the extent of equilibrium swelling of gel by monomer as a function of the degree of crosslinking. ${ }^{7}$ When the gel is a microsphere, a curvature effect should be taken into account. ${ }^{8}$ It is speculated that larger gel spheres can include a large amount of monomer if the other factors are kept unchanged. The equilibrated amount of monomer absorbed in a series of gel particles, obtained by precipitation polymerization or the following seeded polymerization, was measured by adding a defined amount of monomer to the dispersion of $0.2 \mathrm{~g}$ particles in $100 \mathrm{~g}$ water and keeping it at $70^{\circ} \mathrm{C}$ for $1 \mathrm{~h}$ with gentle stirring. Differing from the expectation mentioned above, the extent of equilibrium absorption was almost unchanged and about $9 \%$ of added monomer was absorbed in $0.2 \mathrm{~g}$ gel particles and $91 \%$ remained in $100 \mathrm{~g}$ water regardless of the size of gel particles. No dependence of the extent of absorption on the particle size was attributed to increasing entanglement of interpenetrating polymer chains in the course of repetitive seeded polymerizations. To promote further steps of seeded polymerization, some gradual change of crosslinker/monomer ratio in the charged monomer mixture or addition of some swelling reagents will be necessary.

Precipitation Polymerization in Ethanol/Water As mentioned above, repetive seeded po- 


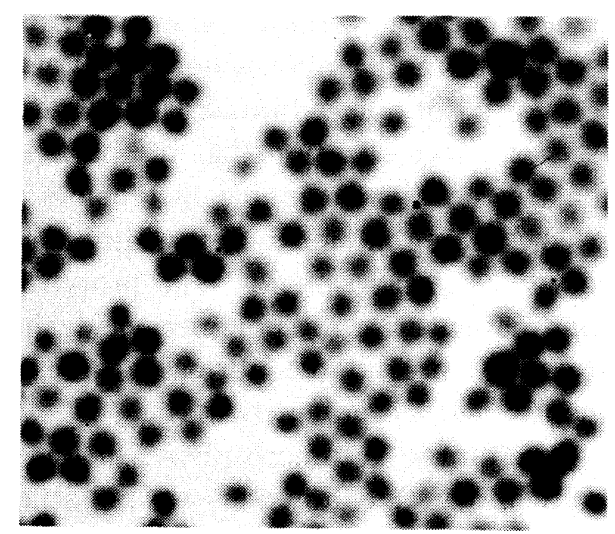

5 a $(0.58 / 1.37)$

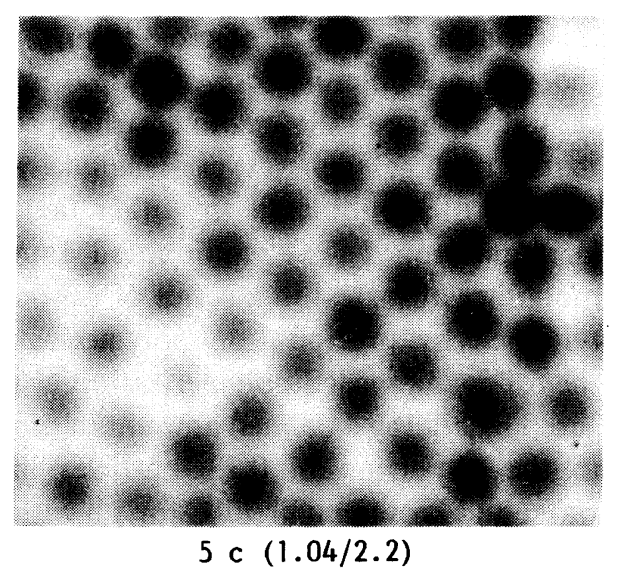

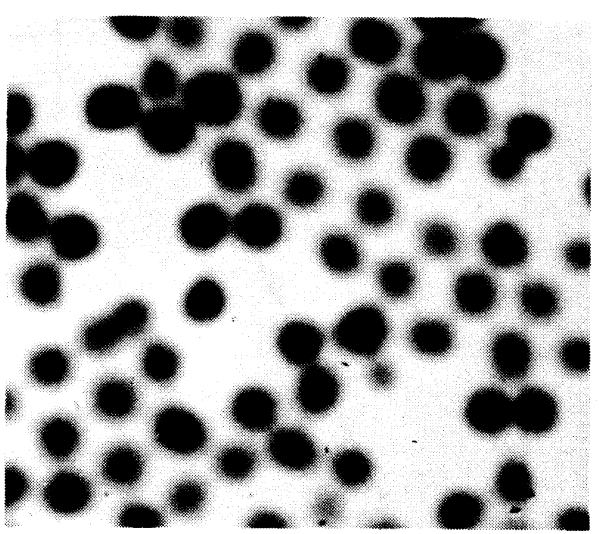

5 b $(0.80 / 2.08)$

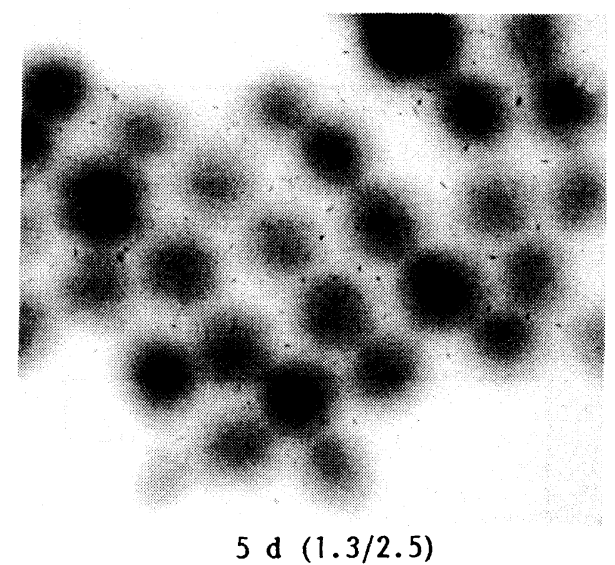

$\stackrel{\min }{\longrightarrow}$

Figure 5. Transmission electron micrographs of poly(acryloyl pyrrolidine) microspheres prepared by precipitation polymerization in the presence of ethanol. APr $/ \mathrm{MBAAm} / \mathrm{APH} /($ water + ethanol $)=1 / 0.1 /$ $0.01 / 200$ in gram, ethanol in medium: $5 \mathrm{a}, 1 \% ; 5 \mathrm{~b}, 2 \% ; 5 \mathrm{c}, 3 \% ; 5 \mathrm{~d}, 4 \% .(\mathrm{m} / \mathrm{n})$ : (diameter of dried particle/ hydrodynamic diameter $(\mu \mathrm{m})$.

lymerizations are somewhat troublesome and inefficient for the preparation of large particles. An alternative method is the precipitation polymerization in the medium containing some solvents for the polymer. The addition of a small amount of such solvents in the polymerization medium must suppress the precipitation of polymer, or, in other words, decrease the nucleation of particles and give a small number of large particles. Ethanol is a good solvent for poly-APr regardless of the temperature. Therefore, the precipitation polymerization of APr was carried out in the presence of 1 to $5 \%$ ethanol and the resulting particles are shown in Figure 5. As expected, ethanol contributed to the formation of large particles very effectively.

It is worth mentioning that the polymerization of APr in ethanol/water with a decreased amount of MBAAm resulted in the formation of viscous solution although this was not the case in the system containing no 
alcohol. Even if a standard amount of MBAAm was employed, the addition of ethanol by more than $5 \%$ gave the similar products. The formation of viscous solution must come from the loose or spread conformation of polymer chains.

The diameter of $1.5 \mu \mathrm{m}$ in the dry state and $2.8 \mu \mathrm{m}$ in the hydrodynamic state was the maximum attained in this study, which was obtained by combining a precipitation polymerization in an ethanol/water medium and one-step seeded polymerization.

\section{CONCLUSIONS}

As the polymers of acryloyl pyrrolidine and acryloyl piperidine have their lower critical solution temperature at $50^{\circ} \mathrm{C}$ and $5^{\circ} \mathrm{C}$, respectively, in water, the aqueous polymerization of these monomers with a crosslinking reagent at $70^{\circ} \mathrm{C}$ resulted in the formation of fine hydrogel microspheres. Repetitive seeded polymeriza- tions could make the microspheres large step by step, but addition of a small amount of ethanol in the polymerization medium gave larger particles more effectively, while keeping monodispersity.

\section{REFERENCES}

1. T. Tanaka and D. J. Fillmore, J. Chem. Phys., 70, 1214 (1979).

2. J. T. Gerig and D. H. Peyton, Int. J. Biol. Macromol., 7, 145 (1985).

3. Y. Hirose, T. Amiya, Y. Hirokawa, and T. Tanaka, Macromolecules, 20, 1342 (1987).

4. R. H. Pelton and P. Chibante, Colloids Surfaces, 20, 247 (1986).

5. H. Kawaguchi, F. Hoshino, and Y. Ohtsuka, Makromol. Chem., Rapid Commun., 7, 109 (1986).

6. F. Hoshino, T. Fujimoto, H. Kawaguchi, and Y. Ohtsuka, Polym. J., 19, 241 (1987).

7. P. J. Flory, "Principles of Polymer Chemistry," Cornell University Press, Ithaca, N. Y., 1953.

8. M. Morton, S. Kaizerman, and M. W. Alter, J. Colloid Sci., 9, 300 (1954). 